Article

\title{
Deep Learning-Based Automatic Clutter/Interference Detection for HFSWR
}

\author{
Ling Zhang ${ }^{1}$, Wei You ${ }^{1,2}$, Q. M. Jonathan $\mathrm{Wu}^{2}$, Shengbo $\mathrm{Qi}^{1, *}$ and Yonggang Ji ${ }^{3}$ \\ 1 College of Engineering, Ocean University of China, Qingdao 266100, Shandong, China; \\ zhangling@ouc.edu.cn (L.Z.); weiy8897@uwindsor.ca (W.Y.) \\ 2 Department of Electrical and Computer Engineering, University of Windsor, Windsor, ON N9B3P4, Canada; \\ jwu@uwindsor.ca \\ 3 First Institute of Oceanography, State Oceanic Administration, Qingdao 266061, Shandong, China; \\ Jiyonggang@fio.org.cn \\ * Correspondence: qsb@ouc.edu.cn; Tel.: +86-532-66781562
}

Received: 31 July 2018; Accepted: 19 September 2018; Published: 21 September 2018

\begin{abstract}
High-frequency surface wave radar (HFSWR) plays an important role in wide area monitoring of the marine target and the sea state. However, the detection ability of HFSWR is severely limited by the strong clutter and the interference, which are difficult to be detected due to many factors such as random occurrence and complex distribution characteristics. Hence the automatic detection of the clutter and interference is an important step towards extracting them. In this paper, an automatic clutter and interference detection method based on deep learning is proposed to improve the performance of HFSWR. Conventionally, the Range-Doppler (RD) spectrum image processing method requires the target feature extraction including feature design and preselection, which is not only complicated and time-consuming, but the quality of the designed features is bound up with the performance of the algorithm. By analyzing the features of the target, the clutter and the interference in RD spectrum images, a lightweight deep convolutional learning network is established based on a faster region-based convolutional neural networks (Faster R-CNN). By using effective feature extraction combined with a classifier, the clutter and the interference can be automatically detected. Due to the end-to-end architecture and the numerous convolutional features, the deep learning-based method can avoid the difficulty and absence of uniform standard inherent in handcrafted feature design and preselection. Field experimental results show that the Faster R-CNN based method can automatically detect the clutter and interference with decent performance and classify them with high accuracy.
\end{abstract}

Keywords: HFSWR; Range-Doppler spectrum; clutter and interference detection; deep learning; Faster R-CNN

\section{Introduction}

High-frequency surface wave radar (HFSWR) transmits high-frequency electromagnetic waves and receives the backscatter echo based on the mechanism of coastal surface diffraction propagation [1]. By analyzing the received echo, continuous monitoring over a wide range can be achieved, which includes vessels [2] and the low altitude flying objects over the sea [3] as well as the ocean dynamics parameters [4-6]. It is widely used and has become the primary technical means in the field of maritime-state monitoring and target vessel detection.

Since HFSWR has the advantages of all-weather operability, and lower cost and wider range of observation as compared to other monitoring systems, it plays an important role in continuous monitoring of our exclusive economic zone and the sea-state. While the strong clutter and interference 
such as the sea clutter, the ionospheric clutter and the radio frequency interference (RFI) severely limit the detection ability of HFSWR. Hence, the detection and suppression of the clutter [7] and the interference [8,9] is essential to guarantee the performance of HFSWR, the automatic and accurate detection in particular is a prerequisite for the suppression of the clutter. Although the suppression of clutter and interference can be implemented without prior detection, both theoretical and simulation results show that the signal to noise ratio (SNR) of the target also can be obviously reduced after implementing clutter/interference suppression [10]. Hence, it is of great importance to detect whether there is existence of clutter/interference before initiating clutter/interference suppression for reservation of the signal energy and improvement of computation efficiency.

The most common ways to identify and detect the clutter and the interference based on Range-Doppler (RD) spectral image are usually divided into two parts: The first step is to extract features from the images; then use the traditional image segmentation techniques to achieve the goal or use the machine learning which is currently the cutting-edge technology in the field of identifying and detecting objects. Chen et al. [11] used the corresponding analysis and cluster analysis methods to classify interference and clutter in the time and the distance domain. In addition, Jin et al. [12] analyzed three typical features of the sea clutter, namely, the backward propagation coefficient, Bragg resonance phenomenon, and the peak amplitude of the Bragg spectrum. These features were sent to support vector machines (SVMs) to be identified and classified into ground/sea clutter. Li et al. [13] proposed a method using the Otsu algorithm to adaptively choose the threshold and extract the features of clutter in the RD spectrum images according to the regional characteristics. Li and Zeng [14] used Gabor wavelet transform to extract the textural features in the RD spectrum images, which could have better performance on the feature extraction of the clutter edge. In order to extract more effective features, Li et al. [15] integrated those features such as statistical property, Gabor features, image characters, and wavelet transform into a feature library, and then processed them using a genetic algorithm to select the better features, which were finally sent to the SVM.

Whether a method was based on traditional image segmentation or on machine learning, these methods relied heavily on artificial feature design and preselection, and hence, human intervention in these methods could not be avoided. Hence, to overcome the above difficulties, an automatic clutter and interference detection method based on deep learning network is proposed.

In 2012, Krizhevsky designed a kind of convolutional neural network (CNN) called the AlexNet [16], which won the image classification competition of ImageNet Large Scale Visual Recognition Competition (ILSVRC). Since the coming of AlexNet, a number of architectures based on CNN, such as Zeiler and Fergus model (ZF) [17] and VGG-16 [18] have been proposed to solve the problems connected with regression and classification. In 2015, Ren et al. proposed the Faster R-CNN [19] and won many titles, including target identification and detection in the ILSVRC and Common Objects in Context (COCO). With this model becoming popular, it has been applied in many image and video processing fields. Compared with the conventional image processing methods, one of the advantages of the $\mathrm{CNN}$ is that it can perform effective feature extraction of the images via the convolutional computation without threshold selection, therefore, it has a wider scope for application. Through its use, the drawbacks caused by the technique of artificial feature design can be totally avoided.

The deep learning method is conventionally designed for big data [20,21], while the specific data with clutter and interference are limited in practice. Based on the distribution of the clutter/interference and the structure of R-CNN, we propose a novel algorithm to identify and detect the clutter and interference based on Faster R-CNN with a new structure of $\mathrm{CNN}$ which has much fewer parameters to be regulated for small datasets. Thanks to the structure of the end-to-end deep $\mathrm{CNN}$, there is no need to design artificial features. Furthermore, the network is able to locate the clutter and interferences, which is helpful for their extraction. Once the clutter and interferences are automatically detected, the suppression algorithm can be started. 
This paper is organized as follows. In Section 2, by analyzing the characteristics of the RD spectrum of HFSWR and the deep learning network, the sea clutter and interference detection problem is formulated via Faster R-CNN in the absence of large amount of required data. In Section 3, we propose a novel detection method based on Faster R-CNN by designing appropriate training and scoring mechanism. In Section 4, field data experiment results are presented to show the effectiveness of the proposed method. In Section 5, the results are discussed, and the future work is highlighted. Finally, the paper is summarized in the concluding remarks in Section 6.

\section{Problem Formulation}

It is recognized that the deep learning networks usually need a large quantity of data. Since HFSWR data is not so massive and requires online, real-time processing, a faster R-CNN, with simple architecture and fewer parameters is needed.

\subsection{Faster $\mathrm{R}-\mathrm{CNN}$}

Faster R-CNN, a real-time object detection network, has a composite architecture which involves the region proposal network (RPN) and Fast R-CNN. This composite architecture is logical because while the former module generates region proposals, the latter detects objects. Due to the "Attention" mechanism, the RPN generating the suggested area frame tells the R-CNN to detect. Furthermore, these two modules share their convolutional layers, which reduces the computational cost of generating region proposals, also the burden on the GPU.

\subsection{Architecture}

For a better learning system, a two-stage four-step architecture is selected. This can be depicted as follows.

- $\quad$ we use the pretrained model to finetune the RPN module for region proposal task;

- we use those region proposals to train the Fast R-CNN, which is also pretrained by the same model for detection task;

- we fix the parameters in the convolutional layers and modulate the second RPN after initializing it by the above detection module, in which the sharing of convolutional computation is completed;

- we repeat step 3 but finetune the parameters, especially those belonging to the second Fast R-CNN.

\subsection{Create a Convolution Neural Network}

When this composite architecture was first proposed, the ZF and VGG-16 networks were chosen to be its pretrained model because these two networks separately contain five shared convolutional layers and 13 shared layers. These deep CNN models are suitable for processing some big datasets like Pascal VOC 2007/2012 or MS COCO (the quantity reaches to $10^{4} \sim 10^{5}$ ), but they are too complicated for those specific fields where a few image samples can be gathered. Therefore, as the RD spectrum data was not large, we create a deep CNN with a relatively small number of parameters to avoid the disadvantages arising from the mismatch between the complexity of the networks and the small dataset.

The whole simple structure, shown in Table 1 includes two convolutional layers and a max pooling layer and two fully connected layers. For detection tasks, the CNN needs to analyze smaller sections of the image, and we should also take into account the amount of spatial detail the CNN needs to resolve into conditions, therefore, we select an input size of $(32 \times 32)$. The reason why we choose two convolutional layers with a small kernel [33] as the core building blocks of CNN is also considered in two parts: The dataset of RD spectrum images we collect is small, and the distinction between the RoIs and the background varies only slightly, hence these two convolutional layers are quite enough for our purpose. The first convolutional layer close to the input layer describes the basic features such as borders, shades and simple stripes, while the second layer contains more abstract and practical 
features, which can possibly decide whether the predication is true or not. The output neurons of these two fully connected layers were set to be as small as possible to subtract the parameters of each fully connected layer, which may account for the majority of the parameters of the whole network.

Table 1. Whole CNN Structure of Faster R-CNN Model.

\begin{tabular}{|c|c|c|}
\hline Layer & Layer Name & Specific Operation \\
\hline 1 & Image Input & $32 \times 32 \times 3$ images with zerocenter normalization \\
\hline 2 & Convolution & $\begin{array}{l}32 \times 3 \times 3 \text { convolutions with stride }\left[\begin{array}{ll}1 & 1\end{array}\right] \text { and } \\
\text { padding }\left[\begin{array}{llll}1 & 1 & 1 & 1\end{array}\right]\end{array}$ \\
\hline 3 & ReLu & ReLu \\
\hline 4 & Convolution & $\begin{array}{l}32 \times 3 \times 3 \text { convolutions with stride }\left[\begin{array}{ll}1 & 1\end{array}\right] \text { and } \\
\text { padding }\left[\begin{array}{cccc}1 & 1 & 1 & 1\end{array}\right]\end{array}$ \\
\hline 5 & ReLu & ReLu \\
\hline 6 & Maxpooling & $\begin{array}{l}3 \times 3 \text { max pooling with stride }\left[\begin{array}{lll}2 & 2\end{array}\right] \text { and } \\
\text { padding }\left[\begin{array}{llll}0 & 0 & 0 & 0\end{array}\right]\end{array}$ \\
\hline 7 & Fully Connected & 64 fully connected layers \\
\hline 8 & ReLu & ReLu \\
\hline 9 & Fully Connected & 2 fully connected layers \\
\hline 10 & Softmax & Softmax \\
\hline 11 & Classification Output & Crossentropyex \\
\hline
\end{tabular}

According to the structure shown in Table 1, we can easily figure out all the parameters of the whole CNN network, which are at the most of $3000(320+320+64+2112+130)$. Compared with the parameter quantity of more than 60 million for the pretrained ImageNet model of AlexNet, our CNN structure is well adapted for processing the small dataset of RD spectrum images.

From the whole CNN structure of the R-CNN model shown in Table 2, we can easily see that the number of parameters in these two models is small. However, Faster R-CNN model spends much less time on the region proposal and the entire process than R-CNN mainly because 1. the R-CNN model does not share convolutional weights between region proposal and SVM classification; and 2. the way that Selective Search as its region proposal method is chosen is much expensive and numerous.

Table 2. Whole CNN Structure of R-CNN Model.

\begin{tabular}{|c|c|c|}
\hline Layer & Layer Name & Specific Operation \\
\hline 1 & Image Input & $32 \times 32 \times 3$ images with zerocenter normalization \\
\hline 2 & Convolution & $32 \times 5 \times 5$ convolutions with stride $\left[\begin{array}{ll}1 & 1\end{array}\right]$ and padding [ 2 \\
\hline 3 & ReLU & ReLU \\
\hline 4 & Max Pooling & $3 \times 3$ max pooling with stride $\left[\begin{array}{ll}2 & 2\end{array}\right]$ and padding $\left[\begin{array}{llll}0 & 0 & 0 & 0\end{array}\right]$ \\
\hline 5 & Convolution & $32 \times 5 \times 5$ convolutions with stride $\left[\begin{array}{ll}1 & 1\end{array}\right]$ and padding $\left[\begin{array}{lll}2 & 2 & 2\end{array}\right.$ \\
\hline 6 & ReLU & ReLU \\
\hline 7 & Max Pooling & $3 \times 3$ max pooling with stride $\left[\begin{array}{ll}2 & 2\end{array}\right]$ and padding $\left[\begin{array}{llll}0 & 0 & 0 & 0\end{array}\right]$ \\
\hline 8 & Convolution & $64 \times 5 \times 5$ convolutions with stride $\left[\begin{array}{ll}1 & 1\end{array}\right]$ and padding [ $\left[\begin{array}{lll}2 & 2 & 2\end{array}\right]$ \\
\hline 9 & ReLU & ReLU \\
\hline 10 & Max Pooling & $3 \times 3$ max pooling with stride $\left[\begin{array}{ll}2 & 2\end{array}\right]$ and padding $\left[\begin{array}{llll}0 & 0 & 0 & 0\end{array}\right]$ \\
\hline 11 & Fully Connected & 64 fully connected layers \\
\hline 12 & ReLU & ReLU \\
\hline 13 & Fully Connected & 10 fully connected layers \\
\hline 14 & Softmax & Softmax \\
\hline 15 & Classification Output & Crossentropyex \\
\hline
\end{tabular}

\section{Detection Method Based on Faster R-CNN}

In this section, the Faster R-CNN-based clutter and interference detection scheme is designed by processing RD spectrum images. To reduce the computation cost, the images are grayscale processed and cropped to a standard size. The gray processing converts the pixel matrix of an image from three channels of RGB into one channel, which helps reduce nearly by $2 / 3$ the cost of the convolutional 
computation between the input layer and the conv1 layer. We then crop the image for cutting down the number of the pixels which also helps reduce the computation cost by $1 / 2$. As the images are preprocessed, we label the region of interests (ROIs) with ground truth boxes and their own titles, such as sea clutter, ground clutter, ionospheric clutter and RFI, which are labeled separately if they appear in the same one image. We show an example in Figure 1 to present the possible region in which clutter and interferences may be localized. Then we send the dataset with the labeled images to the modified Faster R-CNN, which has less convolutional layers than the pretrained ImageNet model. After training, we test the images from the test dataset and analyze the results which contain bounding boxes, labels and scores. The procedure is shown in Figure 2 below. Range means original data done with Fast Fourier Transform (FFT) in distance domain, while range rate means original data done with FFT in Doppler domain.

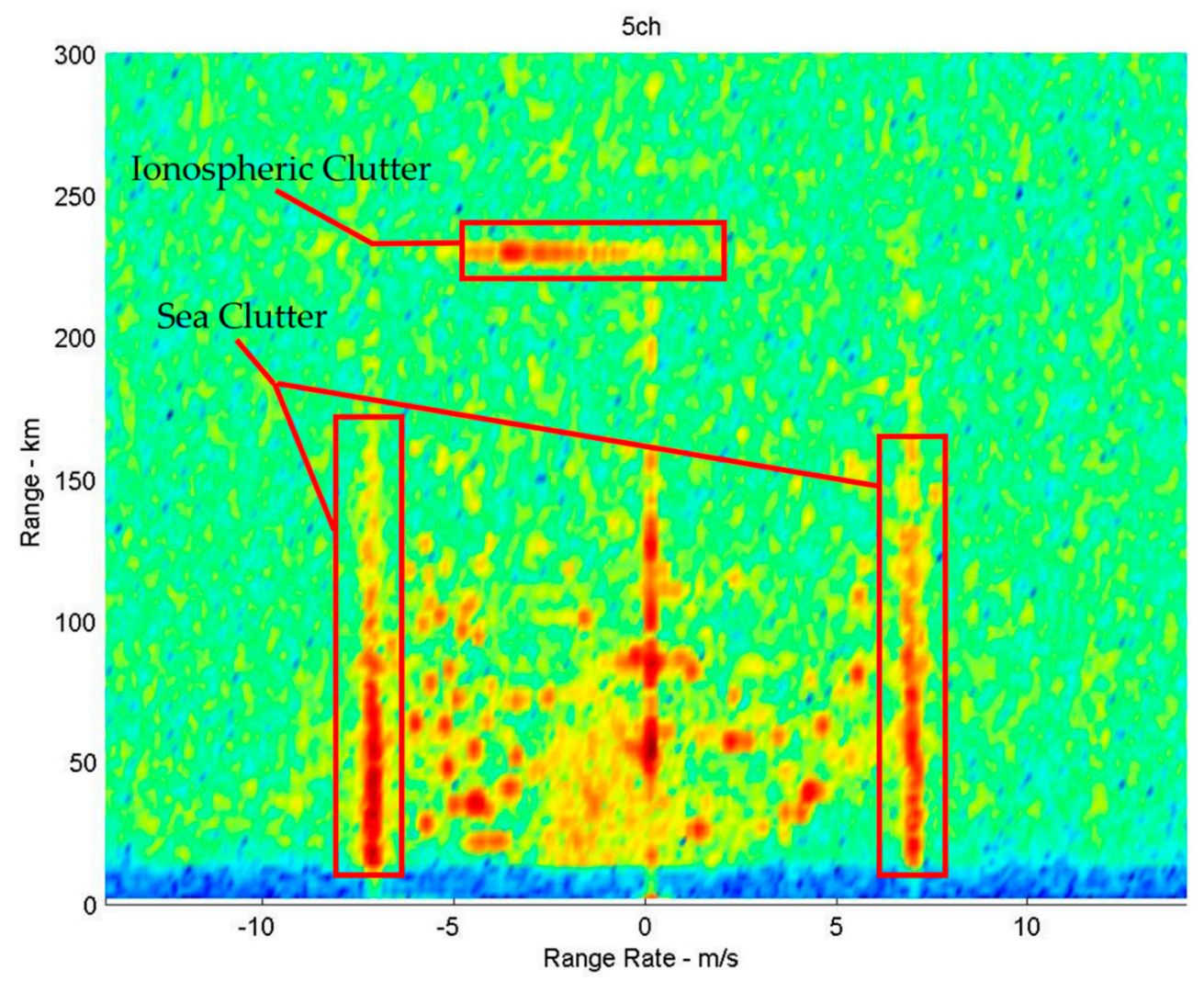

Figure 1. An example of the RD spectrum with labels. (Range means original data done with Fast Fourier Transform (FFT) in distance domain, while range rate means original data done with FFT in doppler domain.).

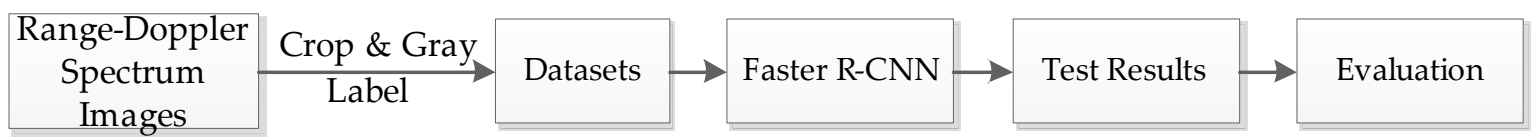

Figure 2. The procedure of the proposed algorithm to detect the clutter and interferences.

\section{Experiments and Results}

\subsection{Dataset}

The three datasets we use to transform into RD spectrum images are all real data recorded from the HFSWR system located in Weihai city. A compact HFSWR with a small array named CORMS (Compact Over-horizon Radar for Marine Surveillance) was used [22]. The first one of the three datasets does not contain RFI, which is suitable for detection of the sea clutter in our experiment. 
And the last two datasets contain RFI. The smaller one is used to detect RFI, while the larger one is used to do the comparison experiment between the two different models. Although there is no need to refine the size of the input images, for computational convenience we still crop the images to the same size $(656 \times 875)$ after the step of graying. We split these images into two parts, training and test image datasets, in the proportion of seven to three.

\subsection{Specific Process}

We sent these training images into the input layer to train the four-step network. The network we built is an end-to-end architecture so that we need to set several parameters till the accomplishment of the training process. The stochastic gradient descent with momentum (SGDM) was applied to renew the weights of each layer while adding a momentum can accelerate the convergence speed of the weights. As the GPU had high memory storage capacity and the low possibility of weights diverging, we preferred to set the batch size to 128 . The learning rates, $e=10^{-5}$ and $e=10^{-6}$ were separately applied to the first two-step and the second two-step stages of the networks. As we would use the above values to train the network for ten times, it meant epoch $=10$.

The main parameters of HFSWR are as follows: The working frequency is $4.7 \mathrm{MHz}$. The coherent integration time $\Delta T$ of the RD image is $300 \mathrm{~s}$. The transmitted wave form is linear frequency modulated interruptive continuous wave. The RD spectrum can be obtained via solving the range and Doppler of the target from the field HFSWR data.

In the test result shown in Figure 3, the vertical bar region was identified as the sea clutter region. The scores on the top indicated that the network had a good performance in the identification of the clutter. Overall, for the samples in this experiment, we chose the average precision (AP) as the index of judging the performance on classification and regression of the bounding boxes.

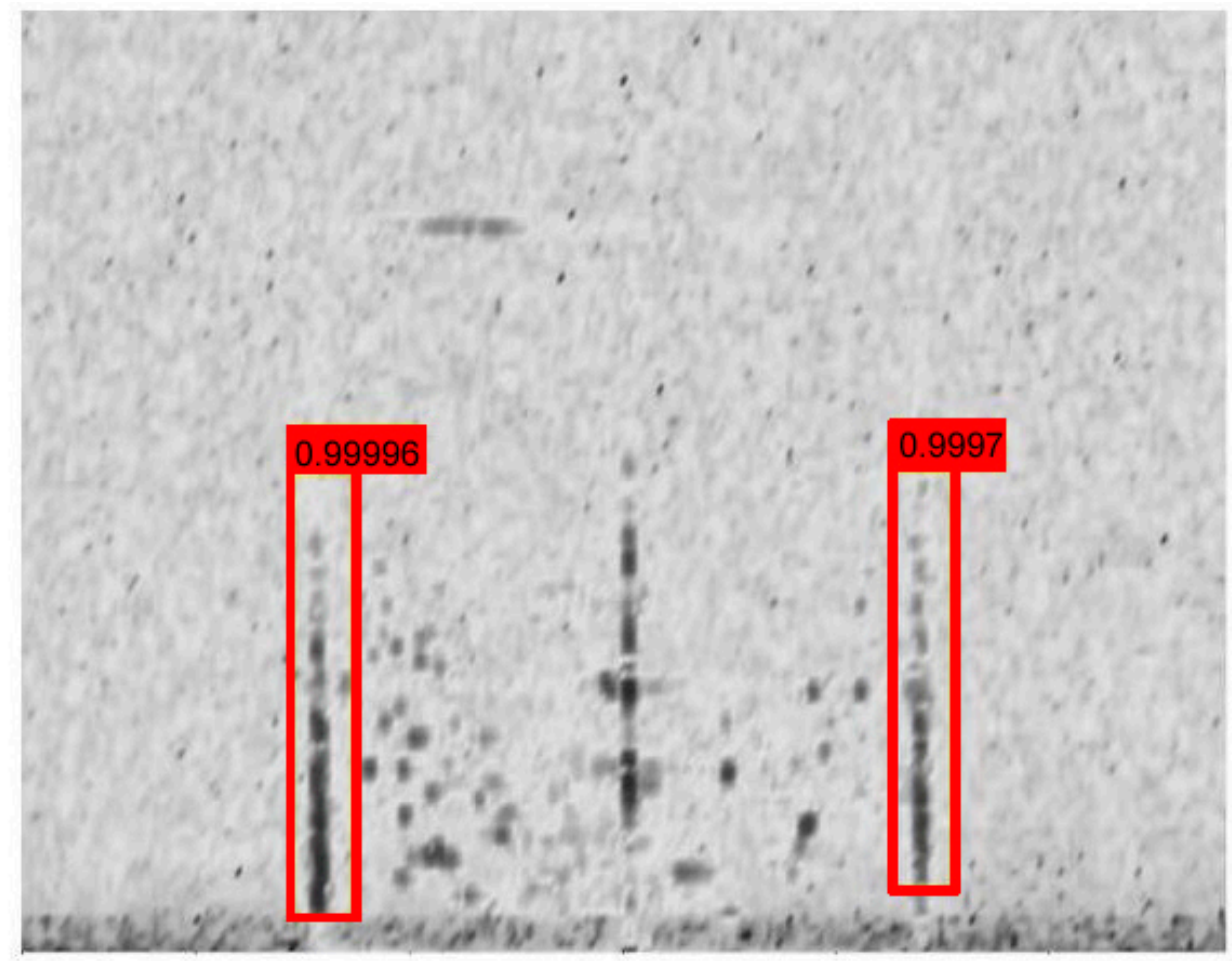

Figure 3. One example of the results of sea clutter detection with the scores.

Recall $=T P /(T P+F N)$, which means the number of the right labeled bounding boxes accounts for the number of the ground truth boxes. Precision $=T P /(T P+F P)$, which means the number of the right labeled bounding boxes accounting for the proportion of the number of all 
the predicted bounding boxes. Under the ideal conditions, these two related indices of Recall and Precision should be both high values.

As shown in Figure 4, when the Recall is 0.94, the Precision can still stay in a high level near 0.96, which verifies the effectiveness of the Faster R-CNN-based clutter detection method as highly accurate. After testing the proposed method over all the samples, the statistical result of AP shows that the proposed method has a high detection accuracy rate of $100 \%$.

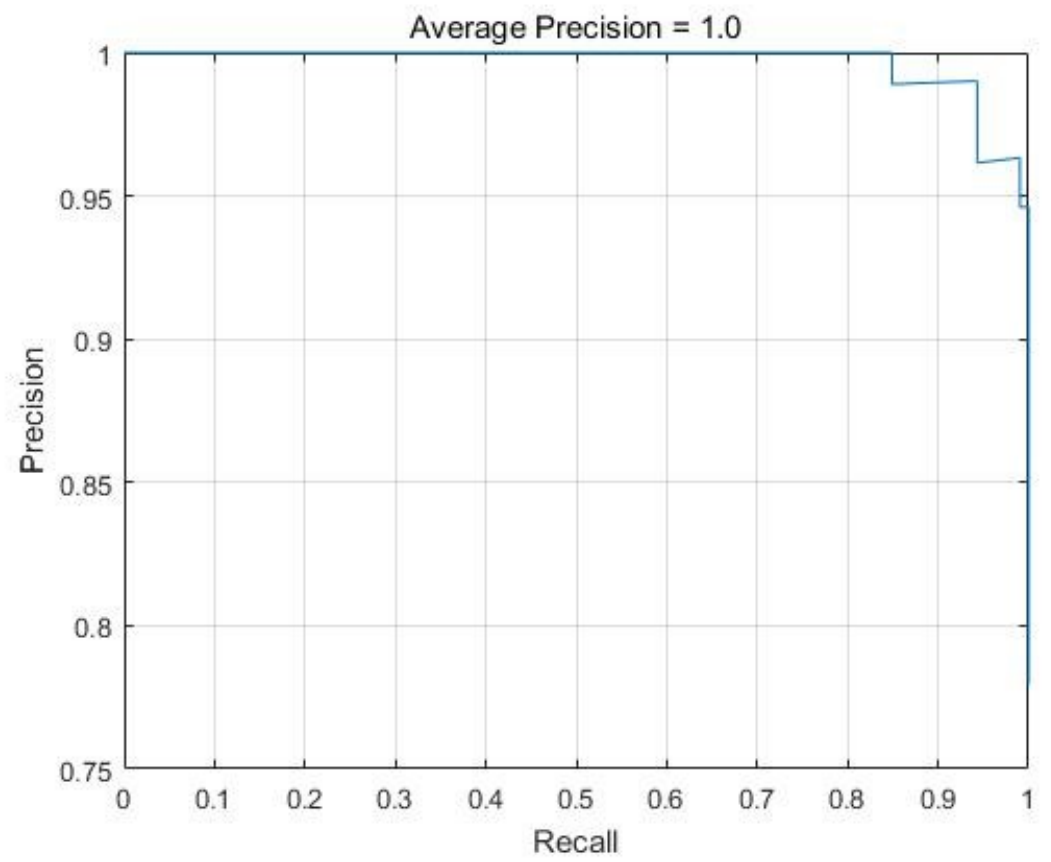

Figure 4. The Precision versus Recall curve of the sea clutter detection in Faster R-CNN.

Moreover, we carried out some experiments on interference detection. We got 200 training images and 40 testing images and set the parameters as for the previous experiment. Figure 5a shows the original RD spectrum with RFI distributed in vertical bar region and Figure 5b illustrates the RFI detection result based on the deep learning network. As an indispensable step in the practical procedure of real-time HFSWR data processing, once the RFI is detected, the suppression method would be initiated to extract RFI automatically, which can maintain the strength of the target in the RD spectrum and reduce the computational cost in comparison with the conventional scheme.

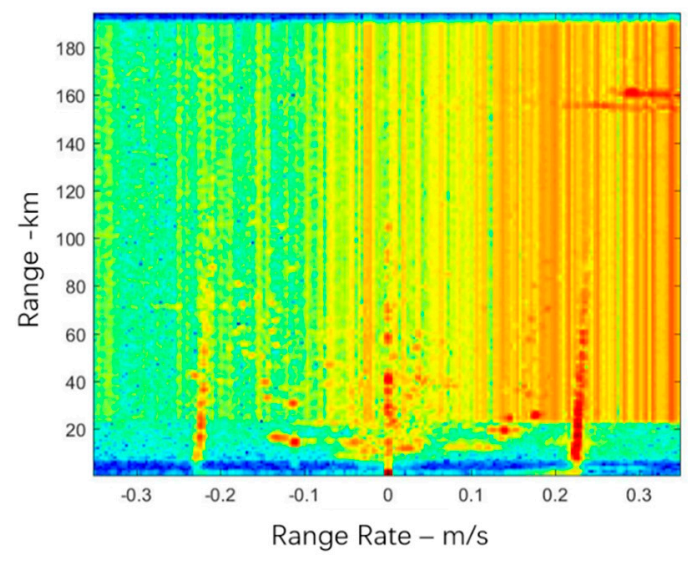

(a)

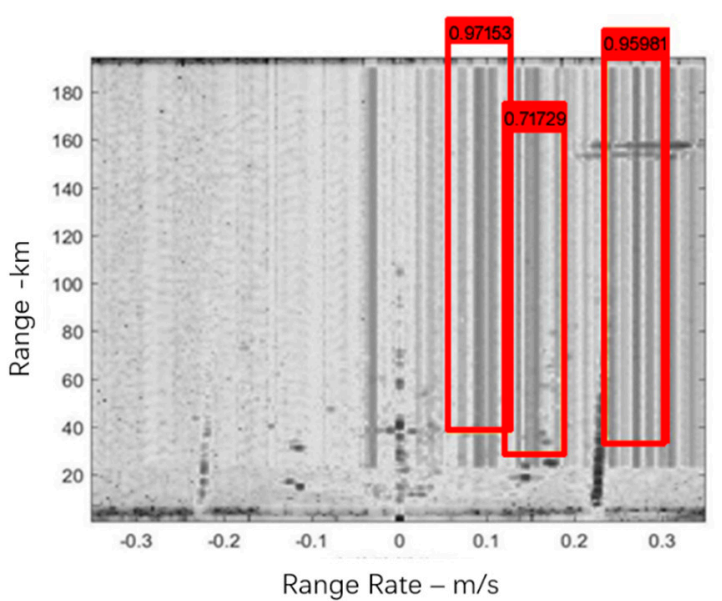

(b)

Figure 5. The original RD spectrum images with RFI (a) and the detection results (b). 


\subsection{Comparison}

In the last series of dataset, we had 410 images which contained all the three pollutions. All the images contained sea clutter, probably mixed with complex RFI or ionospheric clutter. We separately sent them into R-CNN [23] and Faster R-CNN model. In Faster R-CNN model, the speed of testing process reached $0.7 \mathrm{~s}$ per image. In our field-data processing, as the acceptable computation time that satisfies the real-time tracking requirements is below $20 \mathrm{~s}$, the processing time of our method at $0.7 \mathrm{~s}$, means that our model could be used in practical application and would completely solve the problem of real-time processing the HFSWR field data.

\subsubsection{R-CNN}

As R-CNN is the most primitive model used for detection tasks, we chose it for the experimental comparison to highlight the benefits of the region proposals algorithm, which can produce fewer but better region proposals. R-CNN was more representative to be chosen as a comparison model because we can regard R-CNN as a part of its latest incarnation, the Faster R-CNN.

The main structure of R-CNN, shown in Table 2, contains a series of convolutional layers. These CNN layers are pretrained to classify the dataset called CIFAR-10. During the training, we try to keep the parameters which are shared between these two networks unchanged.

\subsubsection{Classification}

Due to the complexity of this dataset, we firstly discuss the classification performance of these two models. As shown in Table 3, we can easily conclude that there is not much difference between these two models in these three classification objects except the RFI. In general, the classification results of Faster R-CNN should be better than those of R-CNN, or at least they should be the same. We deduce that the cause could be the unequal and complex distribution of these pollutions. For example, RFI in these images can have several shapes.

Table 3. Test Results of R-CNN and Faster R-CNN in RFI, Sea Clutter and Ionospheric Clutter Classification.

\begin{tabular}{ccccccccc}
\hline Feature Name. & Model & TP & TN & FP & FN & Precision & Recall & Accuracy \\
\hline RFI & R-CNN & 102 & - & - & 22 & 1.0 & 0.8226 & 0.8226 \\
RFI & Faster R-CNN & 78 & - & - & 46 & 1.0 & 0.6290 & 0.6290 \\
seaClutter & R-CNN & 124 & - & - & - & 1.0 & 1.0 & 1.0 \\
seaClutter & Faster R-CNN & 124 & - & - & - & 1.0 & 1.0 & 1.0 \\
ioClutter & R-CNN & 122 & - & 2 & - & 0.9839 & 1.0 & 0.9839 \\
ioClutter & Faster R-CNN & 122 & - & 2 & - & 0.9839 & 1.0 & 0.9839 \\
\hline
\end{tabular}

\subsubsection{Detection}

But when it comes to the performance in detection, Faster R-CNN is much better than R-CNN as shown in Figure 6 . We can see in Figure $6 \mathrm{c}, \mathrm{f}, \mathrm{i}$ the detection results of R-CNN on the ionospheric clutter, RFI and sea clutter. They are unreasonable and unacceptable. In contrast, the detection results of Faster R-CNN shown in Figure 6b,e,h are much closer to the ground truth. Even the training dataset was much more complex than the two test datasets in the above experiments.

When we discuss the AP shown in Table 4, it seems that neither Faster R-CNN, of which the Precision and Recall Curves are shown in Figure 7, nor R-CNN give good results. From Table 4, we can see clearly that the detection of model R-CNN does not present a decent bounding box that approaches the ground truth box in each image while we notice that each AP in each feature is zero. Even though Faster R-CNN performed a little bit better than R-CNN in this perspective, Faster R-CNN has a better ability to extract fewer but more useful region proposals and to regress the bounding boxes better, thanks to the RPN subnetwork. Also, it has a better performance even with the complex samples which contain multiple forms of RFI. 


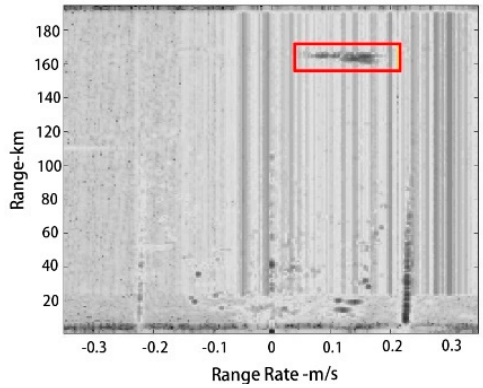

(a)

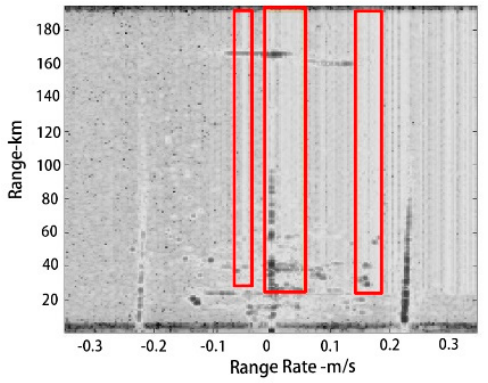

(d)

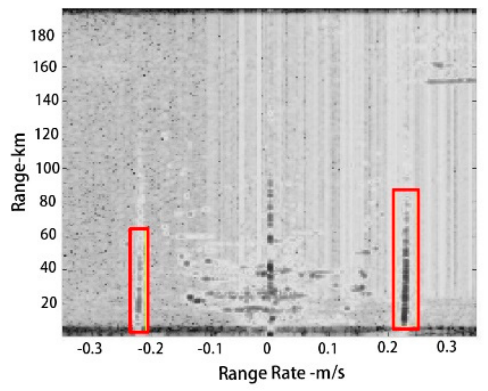

(g)

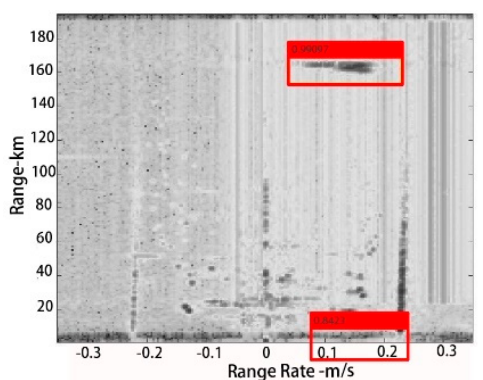

(b)

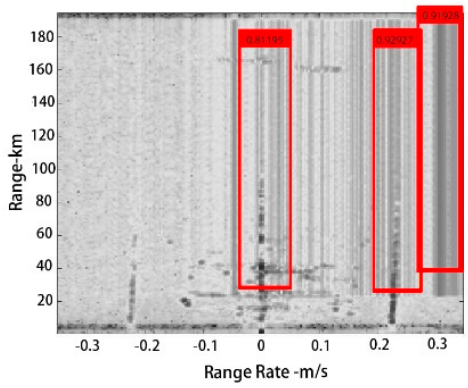

(e)

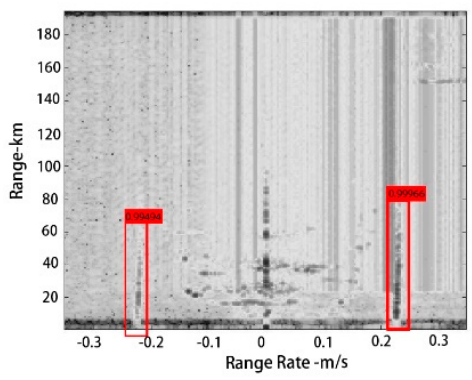

(h)

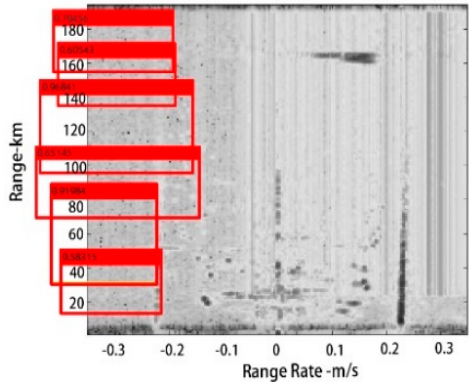

(c)

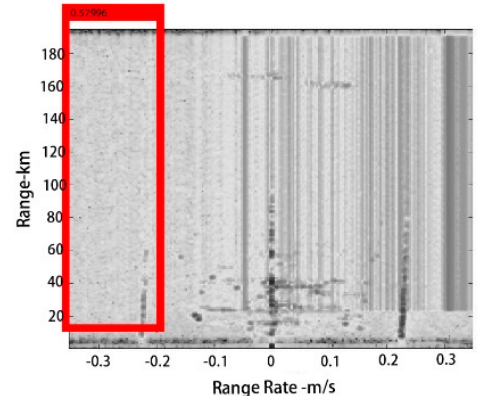

(f)

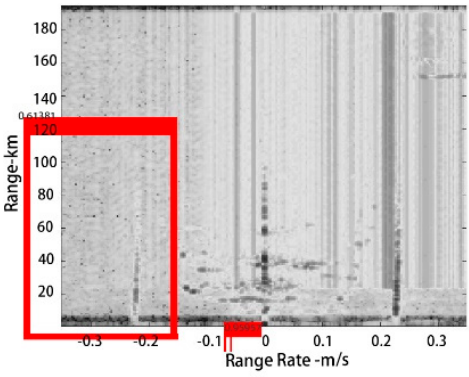

(i)

Figure 6. Comparison between the original images and the Faster R-CNN detection examples as well as the R-CNN detection examples in ionospheric clutter, RFI and sea clutter: $(\mathbf{a}-\mathbf{c})$ show the different detection results in ionospheric clutter; $(\mathbf{d}-\mathbf{f})$ show the different detection results in RFI; $(\mathbf{g}-\mathbf{i})$ show the different detection results in sea clutter.

Table 4. Average Precision of R-CNN and Faster R-CNN in RFI, Ionospheric Clutter and Sea Clutter Detection.

\begin{tabular}{ccc}
\hline Feature Name & Model & AP \\
\hline RFI & R-CNN & 0 \\
RFI & Faster R-CNN & 0.0165 \\
ioClutter & R-CNN & 0 \\
ioClutter & Faster R-CNN & 0.0831 \\
seaClutter & R-CNN & 0 \\
seaClutter & Faster R-CNN & 0.4566 \\
\hline
\end{tabular}

In Figure 7, APs of ionospheric clutter and RFI detection in Faster R-CNN model are both under 0.1 , while the AP of sea clutter is fair enough in this complex samples. We can give an explanation that these samples contain multiple forms of RFI and we do not subdivide them further. In this way, the model cannot distinguish the diversity of RFI. In addition, the distribution of the samples between the training and testing process may be lacking uniformity. For instance, the samples in the training process do not contain much ionospheric clutter but every sample in testing process contains ionospheric clutter. 


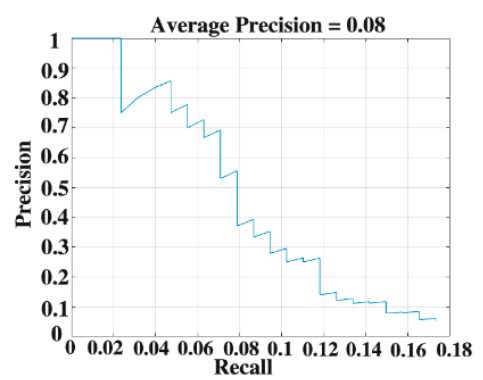

(a)

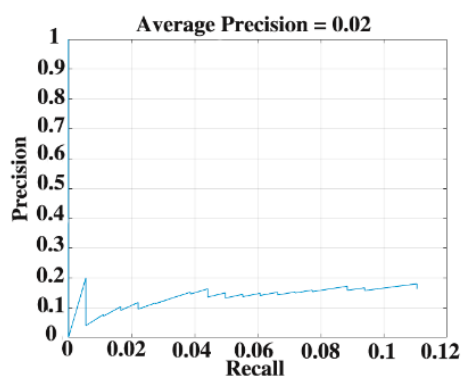

(b)

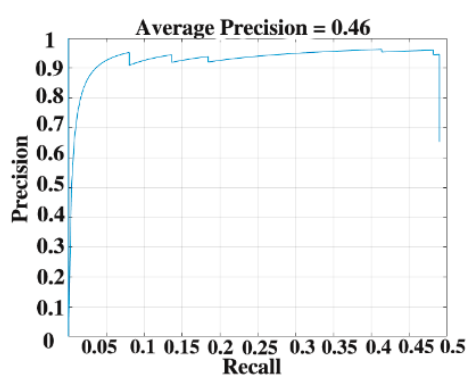

(c)

Figure 7. The three Precision and Recall Curves of ionospheric clutter, RFI and sea clutter tested on Faster R-CNN, showed in sub-figure (a), (b) and (c), respectively.

\section{Discussion}

Of the above three experiments: The first two experiments show how the Faster R-CNN model detects sea clutter and RFI. Notably, the high AP and accurate region proposals both provide the proof that the deep learning method based on the Faster R-CNN model has good detection performance in the field of HFSWR. The last experiment gives a comparison between R-CNN and Faster R-CNN models in a much complex dataset which contains all the three RoIs, namely, sea clutter, ionospheric clutter and multiple forms of RFI. Compared with the AP results of zero tested by the R-CNN model, the results show the advantage of having fewer but more accurate region proposals, which is the benefit from the subnetwork RPN of the Faster R-CNN model.

It is clear that we still need to consider the distribution of the dataset, such as the roughly equal proportion of positive and negative samples, the subdivision of labels owing to the multiple forms of a feature and their distribution and the uniformity among the training and testing samples. There is no doubt that the network based on deep learning has high detection performance, but for further application, more research is required on the accurate detection of ionospheric clutter and RFI, which are more complicated for dataset collection, ground truth labeling, and training of the deep neural network.

\section{Conclusions}

In this paper, a novel method based on deep learning is proposed for automatic detection of clutter and interference in the RD spectrum images produced by HFSWR. The network generated from Faster R-CNN is a framework with RPN and CNN, and is suitable for application in the clutter and interference detection for HFSWR. Field experimental results show that the proposed method has high efficiency and precision that fully meet the requirement of the automatic and real-time detection in practice. Field experiments on ionospheric clutter, RFI and sea clutter detection show that the proposed method has a classification accuracy of $100 \%$ and a decent detection performance. Compared with the conventional detection methods such as the method based on region extraction and subspace decomposition, the deep learning method, which can detect the clutter and interference intelligently and automatically only using the training result, has a wider scope for application.

Author Contributions: Conceptualization, L.Z.; Methodology, Q.M.J.W., L.Z. and W.Y.; Software, W.Y.; Validation, L.Z. and W.Y.; Formal Analysis, W.Y. and S.Q.; Investigation, W.Y.; Resources, Y.J.; Data Curation, Y.J.; Writing-Original Draft Preparation, L.Z. and W.Y.; Writing-Review \& Editing, Q.M.J.W.; Visualization, W.Y.; Supervision, Q.M.J.W.; Project Administration, Y.J.; Funding Acquisition, Y.J.

Funding: This project is sponsored by National Key R\&D Program of China (No. 2017YFC1405202), National Marine Technology Program for Public Welfare (No. 201505002) and the National Natural Science Foundation of China (No. 41506114 and No. 61671166).

Acknowledgments: The authors would acknowledge Ming Li for his great help in conceiving the paper and Jiong Niu for providing important HFSWR data. 
Conflicts of Interest: The authors declare no conflict of interest. The funders had no role in the design of the study; in the collection, analyses, or interpretation of data; in the writing of the manuscript, and in the decision to publish the results.

\section{References}

1. Ponsford, A.M.; Wang, J. A review of high frequency surface wave radar for detection and tracking of ships. Turk. J. Electr. Eng. Comput. Sci. 2010, 18. [CrossRef]

2. Zhang, L.; Li, M.; Niu, J.; Ji, Y. Ionospheric clutter extraction in HFSWR based on range-doppler spectral image processing. In Proceedings of the 2014 IEEE International Conference on Communication Problem-Solving, Beijing, China, 5-7 October 2014; pp. 615-618.

3. Zhao, K.R.; Zhou, G.J.; Yu, C.J.; Quan, T.F. Target flying mode identification and altitude estimation in Bistatic T/R-R HFSWR. In Proceedings of the 17th International Conference on Information Fusion (FUSION), Salamanca, Spain, 7-10 July 2014; pp. 1-8.

4. Yao, G.W.; Xie, J.H.; Ji, Z.Y.; Sun, M.L. The first-order ocean surface cross section for shipborne HFSWR with rotation motion. In Proceedings of the 2017 IEEE Radar Conference (RadarConf), Seattle, WA, USA, 8-12 May 2017; pp. 0447-0450.

5. Li, R.; Hu, Z.; Hartnett, M. Short-Term forecasting of coastal surface currents using high frequency radar data and artificial neural networks. Remote Sens. 2018, 10, 850.

6. Li, M.; Zhang, L.; Wu, X.B.; Yue, X.C.; Emery, W.J.; Yi, X.Z.; Liu, J.F.; Yang, G.B. Ocean surface current extraction scheme with high-frequency distributed hybrid sky-surface wave radar system. Trans. Geosci. Remote Sens. 2018, 56, 4678-4690. [CrossRef]

7. Liu, Y.G.; Weisberg, R.H.; Merz, C.R. Assessment of CODAR and WERA HF radars in mapping currents on the West Florida Shelf. J. Atmos. Ocean. Technol. 2014, 31, 1363-1382. [CrossRef]

8. Merz, C.R.; Liu, Y.G.; Gurgel, K.W.; Petersen, L.; Weisberg, R.H. Effect of radio frequency interference (RFI) noise energy on WERA performance using the "listen before talk" adaptive noise procedure. Coast. Ocean Obs. Syst. 2015, 229-247. [CrossRef]

9. Zhao, C.; Xie, F.; Zhao, C.; He, C. Radio frequency interference mitigation for high-frequency surface wave radar. IEEE Geosci. Remote Sens. Lett. 2018, 15, 986-990. [CrossRef]

10. Jangal, F.; Saillant, S.; Helier, M. Wavelet contribution to remote sensing of the sea and target detection for a high-frequency surface wave radar. IEEE Geosci. Remote Sens. Lett. 2008, 5, 552-556. [CrossRef]

11. Chen, X.; Wu, X.; Liu, J.; Zhang, L. Interference detection method based on correspondence analysis for high frequency ground wave radar. Chin. J. Radio Sci. 2015, 30, 172-176.

12. Jin, Z.L.; Pan, Q.; Liang, Y.; Cheng, Y.M.; Zhou, W.T. SVM-based land/sea clutter identification with multi-features. In Proceedings of the 31st Chinese Control Conference, Hefei, China, 25-27 July 2012; pp. 3903-3908.

13. Li, Y.; Yang, Q.; Zhang, N. Ship detection with adaptive parameter based on detection background analysis. In Proceedings of the 2009 International Conference on Microwave Technology and Computational Electromagnetics (ICMTCE 2009), Beijing, China, 3-6 November 2009; pp. 486-489.

14. Li, Y.; Zeng, W.J.; Zhang, N.; Tang, W.Y. Gabor feature based ionospheric clutter region extraction in Range-Doppler map. In Proceedings of the 2014 IEEE Antennas and Propagation Society International Symposium (APSURSI), Memphis, TN, USA, 6-11 July 2014; pp. 269-270.

15. Li, Y.; He, M.K.; Zhang, N. An ionospheric clutter recognition method based on machine learning. In Proceedings of the 2017 IEEE International Symposium on Antennas and Propagation \& USNC/UNSI National Radio Science Meeting, San Diego, CA, USA, 9-14 July 2017; pp. 1637-1638.

16. Krizhevsky, A.; Sutskever, I.; Hinton, G.E. ImageNet classification with deep convolutional neural networks. Adv. Neural Inf. Process. Syst. 2012, 1, 1-9. [CrossRef]

17. Zeiler, M.D.; Krishnan, D.; Taylor, G.W.; Fergus, R. Deconvolutional networks. In Proceedings of the 2010 IEEE Computer Society Conference on Computer Vision and Pattern Recognition, San Francisco, CA, USA, 13-18 June 2010; pp. 2528-2535.

18. Karen, S.; Andrew, Z. Very deep convolutional networks for large-scale image recognition. arXiv 2014, arXiv:1409.1556. 
19. Ren, S.Q.; He, K.M.; Girshick, R.; Sun, J. Faster R-CNN: Towards real-time object detection with region proposal networks. IEEE Trans. Pattern Anal. Mach. Int. 2017, 39, 1137-1149. [CrossRef] [PubMed]

20. Zhang, Q.C.; Laurence, T.Y.; Chen, Z.K.; Li, P. A survey on deep learning for big data. Inf. Fusion 2018, 42, 146-157. [CrossRef]

21. Liu, X.Y.; Wang, X.D. LS-decomposition for robust recovery of sensory big data. IEEE Trans. Big Data 2017. [CrossRef]

22. Ji, Y.G.; Zhang, J.; Chu, X.L.; Sun, W.F.; Liu, A.J.; Niu, J.; Yu, C.J.; Li, M.; Wang, Y.M.; Zhang, L. A small array HFSWR system for ship surveillance. In Proceedings of the IET International Radar Conference 2015, Hangzhou, China, 14-16 October 2015; pp. 1-5.

23. Girshick, R.; Donahue, J.; Darrell, T.; Malik, J. Rich feature hierarchies for accurate object detection and semantic segmentation. In Proceedings of the 2014 IEEE Conference on Computer Vision and Pattern Recognition, Columbus, OH, USA, 24-27 June 2014; pp. 580-587.

C 2018 by the authors. Licensee MDPI, Basel, Switzerland. This article is an open access article distributed under the terms and conditions of the Creative Commons Attribution (CC BY) license (http:/ / creativecommons.org/licenses/by/4.0/). 